\title{
Central dogma alchemy
}

\author{
KATARZYNA BANDYRA and BEN LUISI \\ Department of Biochemistry, University of Cambridge, CB2 1GA Cambridge, United Kingdom
}

Reflecting on the results of studies of RNA from the last 20 years, one might gain the impression that RNA could be a protein in clever disguise. For decades, RNA had primarily occupied a place in the central dogma of molecular biology as a transient messenger that passively conveys cellular genetic information in a directional flow that starts with DNA, proceeds through RNA, and finally culminates in protein synthesis. Aside from that fleeting role, RNA was also perceived to make more permanent but passive structural contributions as a scaffold for the ribosome or to present anticodons in tRNA. Although these functions for RNA are certainly not entirely inaccurate, the past two decades have revealed that the nucleic acid plays numerous, information-rich, and versatile roles rivaling those often assigned to proteins. The picture emerging from the key advances in the last two decades is that RNA is a chemist, modulator of gene expression both pre- and post-transcription, component of computational networks, allosteric entity, signaling molecule, and an agent of acquired immunity.

Regarding the features of RNA as a chemist, consider how chemical transformations are powerfully mediated by proteins in life-as-we-know it. But before life became so familiar, some of the key transformations that made life possible in the first place are likely to have been mediated by RNA. The work of the Steitz and Moore groups demonstrated in 2000 that the peptidyl transferase site consists of only ribosomal RNA, implicating the RNA in catalyzing peptide bond formation. More recent crystallographic work from the Steitz laboratory highlights the intricate chemistry of the process, involving channeling of protons in a "wire" supported by entrained water and mostly nucleic acid substituents. These observations put overwhelming stereochemical proof of a hypothesis advanced by Harry Noller and others, based on observations that protein-free ribosome preparations can still catalyze peptidyl transfer, that the ribosome is a ribozyme. Other natural ribozyme activities, such as capacity for phospho-transfer, have been illuminated in the last two decades with crystal structures of self-cleaving RNA from the laboratories of Scott, Pyle, and Doudna and many other groups. Piccirilli, Staley and colleagues have obtained evidence that the RNA in the

Corresponding author: ben@cryst.bioc.cam.ac.uk

Article and publication date are at http://www.rnajournal.org/cgi/doi/ $10.1261 /$ rna.050708.115. Freely available online through the RNA Open Access option. spliceosome likely evolved from an RNA catalyst from the group II self-splicing intron. These advances, together with the discovery and structural characterization of artificially selected RNAs that can act as template-based polymerases, have delivered further support that RNA might have been a precursor of the life-as-we-know it. Admittedly, the chemical transformation of peptidyl and phospho-transfer mediated by RNA do not have the high catalytic powers of typical protein-based enzymes in life-as-we-know it; but still to be explored are the capacity of RNA catalysts with metal cofactors in reactions under the reducing conditions of early life, or in photochemical processes.

Another key protein-like feature of RNA is its capacity to communicate allosteric signals and, perhaps somewhat overlooked, its potential for cooperative behavior. Cooperativity underlies the capacity of modern cells to respond rapidly to changes in environment, or to signal for genetic programs in metazoan embryogenesis, or social behavior in prokaryotes. An important allosteric effect of RNA is the ability to switch conformation upon binding effector molecules or in response to environmental changes, for instance acting as a thermometer with temperature-dependent structural states. The discovery and characterization of metabolite-responsive RNA "riboswitches" that are often a part of an mRNA, by the Breaker group and other laboratories, demonstrates RNA not being a passive carrier but rather an active entity that can change conformation induced by ligand binding, with functional consequences on rates of translation and degradation. Allosteric and cooperative effects play roles in the recruitment of binding partners in ribonucleoprotein complexes such as the splicing machinery, as illuminated by the structural work of Kiyoshi Nagai and others. RNA can also be an allosteric ligand for other RNAs, which in turn can communicate recognition signals for proteins. Given the capacity of RNA to be remodeled with functional consequence through interactions with metabolites, proteins, and other RNA, it is perhaps little wonder that the cells are replete with RNA helicases, which are energy-dependent machines that keep these interactions in a state of flux. Structural and functional studies of helicases over the last two decades suggest that dynamic remodeling might underlie sensitive

(C) 2015 Bandyra and Luisi This article, published in $R N A$, is available under a Creative Commons License (Attribution-NonCommercial 4.0 International), as described at http://creativecommons.org/licenses/by-nc/4.0/. 
responses to environmental conditions mediated by RNA allostery.

The major discoveries in the RNA world over the past 20 years have been dominated by the structure of the ribosome and mechanism of protein translation. These have tremendously expanded the recognized repertoire of RNA secondary and tertiary folds. However, the RNA in the ribosome has turned out to be more than just the skeleton of this ribonucleoprotein; it is now seen to have allosteric behavior that is fundamental for communicating structural signals in almost every key step of protein synthesis. The structural data have illuminated how the cognate pairing of tRNA and mRNA at the codon recognition center triggers a chain of events that faithfully bring the correct amino acid at the peptidyl transfer center in the ribosome through allosteric activation of GTP hydrolysis. Structural deformations in the tRNA and ribosome, visualized by crystallography and cryoEM, are key for maintaining fidelity of translation. The structural and functional work on the ribosome has been a remarkable achievement with international efforts led by the laboratories of Thomas Steitz, Peter Moore, Venki Ramakrishnan, Ada Yonath, Harry Noller, and Marat Yusupov. New discoveries continue to be made through the rapid advances in cryoEM techniques that illuminate the remarkably conserved mechanism of peptide secretion through translocons.

Another remarkable parallel between RNA and proteins, made clear over the last 20 years, is the capacity to participate in elaborate regulatory networks. Transcription factors are proteins that, through DNA binding or covalent modification of chromatin, make intricate and beautifully interconnected patterns that give complex control of gene expression. It is now becoming clear that RNAs can provide the same networking too, post-transcription. With the discovery of microRNAs in the 1990s, a re-examination commenced of short RNA fragments that were previously considered to be just cellular noise. Numerous classes of non-coding RNAs were identified that have key roles in regulation of gene expression in eukaryotes. Moreover, RNA mediated "riboregulation" was also found in bacteria, where it often helps in rapid adaptation to changes in the environment. Another set of key discoveries concerns the contribution of riboregu- lation to pre-transcriptional control, through activation of chromatin modification in promoter regions in eukaryotic genomes, or by regulating polymerase in bacterial (by physical sequestration of 6S RNA). RNA is also capable of modulating protein activities by physical sequestration, for instance in acting as an anti-toxin to a protein toxin.

Many achievements in RNA biology were made possible by the development of RNA sequencing techniques. This led not only to better understanding of some biological processes in the investigated cell, but also to comparison of transcriptomes between, e.g., tissues or cellular lines grown under different conditions. With its widespread use currently, RNA sequencing has contributed to identification of gene expression patterns in response to environmental adaptation or in diseases. It has also proven to be a powerful tool for discovery through applications such as ribosome profiling, which has illuminated numerous features of translation including recognition of regulatory elements and control.

Immunity was long thought to be the exclusive domain of proteins, which can be used to recognize diverse epitopes. In metazoans, viral RNA can be recognized by chemical signatures on the $5^{\prime}$ end and structure, such as recognition by the Rig-I helicase. RNA can thus be a signaling molecule to initiate innate immune response. A surprising discovery was that RNA can recognize foreign nucleic acids and confer acquired immunity against viruses in bacteria. The discovery of this system - the CRISPR system-was soon followed by applications for genome engineering, which is widely exploited in research, and could possibly be applied in medicine.

We are moving from the perception that the information contained in RNA is mainly encoded in its linear sequence, but the emerging image of this molecule, accounting for the numerous roles it can play in the cell, shows that the sequence is only one element of complex entirety. RNA is no longer considered just a string of nucleotides, but rather as a structured molecule capable of catalyzing enzymatic reactions, interacting with and modulating its RNA and protein partners, as well as regulating many physiological processes. It is not only a template for protein synthesis, but protein-like formation, that forms a parallel to proteome network in the cell to help carry out and maintain the life-as-we-know it. 

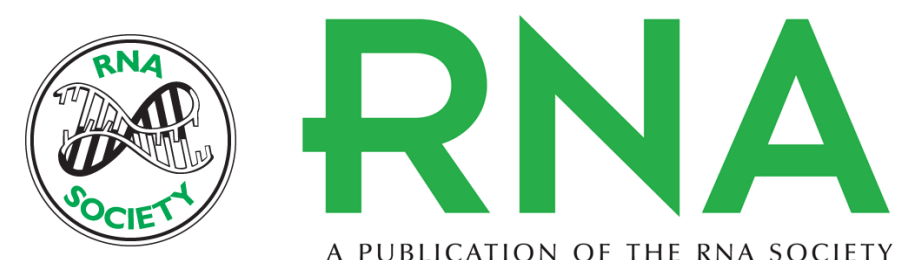

A PUBLICATION OF THE RNA SOCIETY

\section{Central dogma alchemy}

Katarzyna Bandyra and Ben Luisi

RNA 2015 21: 558-559

Open Access Freely available online through the RNA Open Access option.

Creative This article, published in RNA, is available under a Creative Commons License

Commons (Attribution-NonCommercial 4.0 International), as described at

License http://creativecommons.org/licenses/by-nc/4.0/.

Email Alerting Receive free email alerts when new articles cite this article - sign up in the box at the Service top right corner of the article or click here.

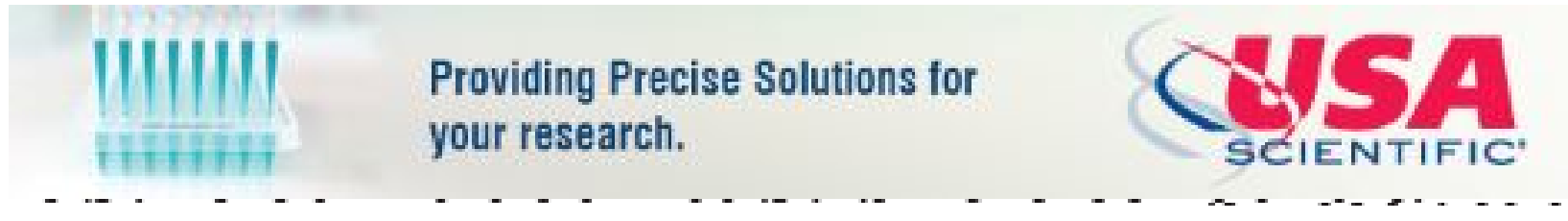

To subscribe to $R N A$ go to:

http://rnajournal.cshlp.org/subscriptions

(C) 2015 Bandyra and Luisi; Published by Cold Spring Harbor Laboratory Press for the RNA Society 